\title{
Epicardial Fat: Can It Be an Easy Screening Tool in Preventive Cardiology to Guide Lifestyle Modifications and Therapy in Coronary Artery Disease in the Developing Countries?
}

\section{Sandeep Kumar Kar*}

Department of Cardiac Anaesthesiology, Institute of Postgraduate Medical Education and Research, Kolkata, India

\section{Introduction}

In the developing countries, the perennial search for an easily available, cheap non-invasive tool for screening high risk patients prone to Major Adverse Cardiac Events (MACE) has always been a heartfelt need in patients with high risk for rapid progression of coronary artery disease. Traditionally Waist to Hip ratio has been used and is quoted in many studies as an epidemiological tool to guide lifestyle modification and therapeutic interventions. Currently Epicardial fat is often in discussion in many forums as a screening tool.

In the adult human heart, Epicardial fat is commonly found in the atrioventricular and interventricular grooves. Minor foci of fat are also located subepicardially along the free walls of the atria and around the two appendages. Increase in the volume of the of the Epicardial fat leads to progressively filling up of the space between the ventricles, at times covering the entire Epicardial surface. A small amount of fat tissue may also extend from the Epicardial surface into the myocardium, often following the adventitia of the branches of the coronary arteries. There is no muscle or fascia dividing Epicardial fat and the myocardium; therefore, these two tissues share the same microcirculation [1].

\section{Functional Significance of Epicardial Fat}

Epicardial fat is highly metabolically active with higher rates of fatty acid uptake and secretion than other visceral fat depots. Under normal physiologic conditions, Epicardial fat serve as a buffer, absorbing fatty acids and protecting the heart against high fatty acids levels., Epicardial fat may serve as an energy store house at times of high metabolic demand, Epicardial fat channels fatty acids to the myocardium [2]; and as brown fat it protects the myocardium from hypothermia [3]. Epicardial fat may release factors that blunt the toxic effects of high fatty acid levels on the myocardium; it may also release factors that promote harmful coronary artery and myocardial changes [2].

A body of evidence shows that Epicardial fat is a metabolically active organ that produces several bioactive adipokines. It is a source of several proinflammatory and proatherogenic cytokines, as well as tumour necrosis factor, monocyte chemoattractant protein-1, interlukin-6, nerve growth factor, resistin, visfatin, omentin, leptin, plasminogen activator inhibitor-1, and angiotensinogen [4-10]. However, Epicardial fat also produces anti-inflammatory, antiatherogenic cardio-protective adipokines, such as adiponectin and adrenomedullin [11-14]. The rate of progression of atheroscreotic disease depends on the relative dominance of the anti-atherosclerotic, or pro-inflammatory and proatherogenic mediators released by epicardial fat tissue. Proximity to the heart and the absence of fascial boundaries, demarcating epicardial fat from the myocardium and, sharing of the common microcirculation between the two tissues may help epicardial adipose tissue interact locally and modulate the coronary arteries through the paracrine or vasocrine secretion of pro-inflammatory adipokines.

\section{Echocardiographic and Computed Tomographic Assessment of Epicardial Fat}

Standard parasternal long-axis and short-axis views from $2 \mathrm{D}$ images permit the most accurate measurement of epicardial fat thickness on the right ventricle, with optimal cursor beam orientation in each view. Echocardiographically, epicardial fat is generally identified as the relatively echo-free space between the outer wall of the myocardium and the visceral layer of pericardium. Its thickness is measured perpendicularly on the free wall of the right ventricle at endsystole in 3 cardiac cycles and then averaged for accurate assessment [15]. Because as right ventricle is compressed during diastole, epicardial fat thickness is best measured at end-systole at the point on the free wall of the right ventricle at which the ultrasound beam is oriented in a perpendicular manner, using the aortic annulus as an anatomic landmark [16]. A normal upper-limit value for epicardial fat thickness has not been established by large scale trials. Echocardiographic epicardial fat thickness varies from a minimum of $1 \mathrm{~mm}$ to a maximum measured value of almost $23 \mathrm{~mm}$ [16]. The wide range of epicardial fat thicknesses likely reflects the substantial variation in abdominal visceral fat distribution. Iacobellis et al. [16] found median epicardial fat thicknesses of $7 \mathrm{~mm}$ in men and $6.5 \mathrm{~mm}$ in women in a large population of patients who underwent transthoracic echocardiography for standard clinical indications.

Echocardiographic epicardial fat measurement may have some advantages as an index of high cardio-metabolic risk and it is better suited to be a screening tool in developing countries because of the following reasons:

1. Being a direct measure of visceral fat rather than an anthropometric measure, such as waist circumference, that includes muscle and skin layers, the echocardiographic measurement of epicardial fat is a more sensitive and specific measure of true visceral fat content.

2. It is an objective, non-invasive, readily available, and less expensive measure of visceral fat than MRI or CT, ideally suited for a resource poor setting as encountered in health care delivery systems in developing countries.

*Corresponding author: Dr. Sandeep Kumar Kar, Assistant Professor, Department of Cardiac Anaesthesiology, Institute of Postgraduate Medical Education and Research, Kolkata, India, Tel: 09477234900; E-mail: sndpkar@yahoo.co.in

Received March 20, 2017; Accepted March 21, 2017; Published March 27, 2017

Citation: Kar SK (2017) Epicardial Fat: Can It Be an Easy Screening Tool in Preventive Cardiology to Guide Lifestyle Modifications and Therapy in Coronary Artery Disease in the Developing Countries? J Bioanal Biomed 9: e150. doi: 10.4172/1948-593X.1000e150

Copyright: (c) 2017 Kar SK. This is an open-access article distributed under the terms of the Creative Commons Attribution License, which permits unrestricted use, distribution, and reproduction in any medium, provided the original author and source are credited. 
3. Visceral cardiac fat can be quantified fairly precisely compared with ectopic fat deposition in organs such as the liver, which can be described only qualitatively unless expensive accurate measurements are made, such as CT or MRI.

4. Measured echocardiographic epicardial fat is a direct measure of ectopic fat deposition, whereas anthropometric measures can be associated only with ectopic fat deposition.

5. It can be measured even from echocardiograms that were not specifically performed to optimize the measurement of epicardial fat.

6. It can be compared with other echocardiographic parameters, such LV mass and ejection fraction, traditionally associated with cardiovascular risk.

7. Echocardiographic epicardial fat could be a more reliable quantitative therapeutic marker during interventions modulating and reducing visceral adiposity and thus guide weight loss programs and anti-lipedemic therapy for hyper-lipidemia.

An advantage of CT based epicardial fat estimation is that it can be readily combined with coronary calcium scoring or CT coronary angiography. MRI is the current gold standard for quantitative estimation of epicardial fat, it has the advantage of being radiation free but it is expensive and not widely available. So it cannot be an ideal screening tool in health care and delivery system in resource limited, developing countries.

\section{Epicardial Fat as a Tool to Guide Life Style Modification in Preventive Cardiology}

On the basis of the evidence that epicardial fat reflects visceral adiposity, its echocardiographic measurement has been used as a therapeutic target in subjects who undergo weight-loss interventions [17], bariatric surgery [18], exercise programs [19], and hormonereplacement treatment [20].

Echocardiographic epicardial fat thickness significantly decreased in all 3 studies that included body weight modulations and changes. In fact, epicardial fat decreased after very low calorie diet, bariatric surgery-induced weight loss, and moderate aerobic exercise.

The weight loss intervention study showed that the decrease in epicardial fat during weight loss was quicker and higher than the decreases in body mass index, waist circumference, and body weight, common indices of body fatness [17]. It can be assumed that epicardial fat reflects the more rapid and massive visceral fat loss after a very low calorie diet. This finding may open new options in the management of patients with high cardio-metabolic risk and metabolic syndrome.

\section{Epicardial Fat as a Target for Therapy}

Weight loss attained by patients undergoing weight loss programs is accompanied by rapid loss of epicardial fat from their myocardium, the rationality for this finding being, epicardial fat is a direct measure of total visceral adiposity, Reduction in one is bound to affect the other as they are in a close metabolic association [17]. Statins, Ezetimibe and certain anti- diabetic drugs reduce epicardial fat volume, however most dramatic and consistent reduction in epicardial fat volume is achieved by atorvastatin, in comparison to simvastatin, pravastatin and Ezetimibe [21].

Anti-diabetic drugs like pioglitazone, glucagon type peptide 1 agonists (liraglutide, exenatide) and dipeptidyl peptidase-4 inhibitors
(Sitaglipin) have been found to reduce the volume of epicardial fat as well as the level of proinflammatory and atherogenic markers secreted by the metabolically active epicardial fat tissue [22]. Lastly Human growth hormone therapies also reduce echocardiographically visible epicardial fat thickness [20].

\section{Conclusion}

Epicardial fat assessment by echocardiography can serve as a cheap screening tool in developing countries as it is non-invasive, easy to perform, valid and reproducible. This can very well guide modification in life style measures, weight loss programs as well as serve as an assessment tool to measure the response to therapy (both anti-lipidemic and anti-diabetic drugs) and thereby target risk reduction in coronary artery disease.

\section{References}

1. lacobellis G, Corradi D, Sharma AM (2005) Epicardial adipose tissue: anatomic biomolecular and clinical relationships with the heart. Nat Clin Pract Cardiovasc Med 2: 536-543.

2. Marchington JM, Pond CM (1990) Site specific properties of pericardia and epicardial adipose tissue: the effects of insulin and high-fat feeding on lipogenesis and the incorporation of fatty acids in vivo. Int J Obes 14: 10131022.

3. Sacks HS, Fain JN, Holman B, Cheema P, Chary A, et al. (2009) Uncoupling protein-1 and related mRNAs in human epicardial and other adipose tissues: epicardial fat functioning as brown fat. J Clin Endocrinol Metab 94: 3611-3615.

4. Mazurek T, Zhang L, Zalewski A, Mannion JD, Diehl JT, et al. (2003) Human epicardial adipose tissue is a source of inflammatory mediators. Circulation 108: $2460-2466$

5. Sacks HS, Fain JN (2007) Human epicardial adipose tissue: a review. Am Heart J 153: 907-917.

6. Baker AR, Silva NF, Quinn DW, Harte AL, Pagano D, et al. (2006) Human epicardial adipose tissue expresses a pathogenic profile of adipocytokines in patients with cardiovascular disease. Cardiovasc Diabetol 5:1.

7. Chaldakov GN, Fiore M, Stankulov IS, Manni L, Hristova MG, et al. (2004) Neurotrophin presence in human coronary atherosclerosis and metabolic syndrome: a role for NGF and BDNF in cardiovascular disease? Prog Brain Res 146: 279-289.

8. Kremen J, Dolinkova M, Krajickova J, Blaha J, Anderlova K, et al. (2006) Increased subcutaneous and epicardial adipose tissue production of proinflammatory cytokines in cardiac surgery patients: possible role in postoperative insulin resistance. J Clin Endocrinol Metab 91: 4620-4627.

9. Cheng KH, Chu CS, Lee KT, Lin TH, Hsieh CC, et al. (2008) Adipocytokines and proinflammatory mediators from abdominal and epicardial adipose tissue in patients with coronary artery disease. Int J Obes 32: 268-274.

10. Fain JN, Sacks HS, Buehrer B, Bahouth SW, Garrett E, et al. (2008) Identification of omentin mRNA in human epicardial adipose tissue: comparison to omentin in subcutaneous, internal mammary artery periadventitial and visceral abdominal depots. Int J Obes (Lond) 32: 810-815.

11. lacobellis G, Pistilli D, Gucciardo M, Leonetti F, Miraldi F, et al. (2005) Adiponectin expression in human epicardial adipose tissue in vivo is lower in patients with coronary artery disease. Cytokine 29: 251-255.

12. lacobellis G, di Gioia CR, Cotesta D, Petramala L, Travaglini C, et al. (2009) Epicardial adipose tissue adiponectin expression is related to intracoronary adiponectin levels. Horm Metab Res 41: 227-231.

13. Silaghi A, Achard V, Paulmyer-Lacroix O, Scridon T, Tassistro V, et al. (2007) Expression of adrenomedullin in human epicardial adipose tissue: role of coronary status. Am J Physiol Endocrinol Metab 293: E1443-E1450.

14. lacobellis G, Gioia CR, Di Vito M, Petramala L, Cotesta D, et al. Epicardial adipose tissue and intracoronary adrenomedullin levels in coronary artery disease. Horm Metab Res 41: 855-860.

15. lacobellis G, Assael F, Ribaudo MC, Zappaterreno A, Alessi G, et al. (2003) 
Citation: Kar SK (2017) Epicardial Fat: Can It Be an Easy Screening Tool in Preventive Cardiology to Guide Lifestyle Modifications and Therapy in Coronary Artery Disease in the Developing Countries? J Bioanal Biomed 9: e150. doi: 10.4172/1948-593X.1000e150

Epicardial fat from echocardiography: a new method for visceral adipose tissue prediction. Obes Res 11: 304-310.

16. lacobellis G, Willens HJ, Barbaro G, Sharma AM (2008) Threshold values of high risk echocardiographic epicardial fat thickness. Obesity 16: 887-892.

17. lacobellis G, Singh N, Wharton S, Sharma AM (2008) Substantial changes in epicardial fat thickness after weight loss in severely obese subjects. Obesity 16: 1693-1697.

18. Willens HJ, Byers P, Chirinos JA, Labrador E, Hare JM, et al. (2007) Effects of weight loss after bariatric surgery on epicardial fat measured using echocardiography. Am J Cardiol 99: 1242-1245.

19. Kim MK, Tomita T, Kim MJ, Sasai H, Maeda S, et al. (2009) Aerobic exercise training reduces epicardial fat in obese men. J Appl Physiol 106: 5-11.
20. Lanes R, Soros A, Flores K, Gunczler P, Carrillo E, et al. (2005) Endothelial function, carotid artery intima-media thickness, epicardial adipose tissue, and left ventricular mass and function in growth hormone-deficient adolescents: apparent effects of growth hormone treatment on these parameters. J Clin Endocrinol Metab 90: 3978-3982.

21. Park JH, Park YS, Kim YJ (2010) Effects of Statins on the Epicardial Fa Thickness in Patients with Coronary Artery Stenosis Underwent Percutaneous Coronary Intervention: Comparison of Atorvastatin with Simvastatin/Ezetimibe. J Cardiovasc Ultrasound 18: 121-126.

22. Lima-Martínez MM, Paoli M, Rodney M, Balladares N, Contreras M, et al (2016) Effect of sitagliptin on epicardial fat thickness in subjects with type 2 diabetes and obesity: a pilot study. Endocrine 51: 448-455 